\title{
Novel transcription-induced fusion RNAs in prostate cancer
}

\author{
Sen Zhao ${ }^{1,2}$, Marthe Løvf ${ }^{1,2}$, Kristina Totland Carm ${ }^{1,2}$, Anne Cathrine Bakken ${ }^{1,2}$, \\ Andreas M. Hoff ${ }^{1,2}$ and Rolf I. Skotheim ${ }^{1,2,3}$ \\ ${ }^{1}$ Department of Molecular Oncology, Institute for Cancer Research, Oslo University Hospital-Norwegian Radium Hospital, \\ Oslo, Norway \\ ${ }^{2}$ Center for Cancer Biomedicine, Faculty of Medicine, University of Oslo, Oslo, Norway \\ ${ }^{3}$ Department of Informatics, Faculty of Natural Science and Mathematics, University of Oslo, Oslo Norway \\ Correspondence to: Rolf I. Skotheim, email: rolf.i.skotheim@rr-research.no
}

Keywords: prostate cancer, fusion transcript, RNA sequencing, expression profile

Received: July 07, $2016 \quad$ Accepted: April 03, $2017 \quad$ Published: April 13, 2017

Copyright: Zhao et al. This is an open-access article distributed under the terms of the Creative Commons Attribution License 3.0 (CC BY 3.0), which permits unrestricted use, distribution, and reproduction in any medium, provided the original author and source are credited.

\section{ABSTRACT}

Prostate cancer is a clinically and pathologically heterogeneous disease with a broad spectrum of molecular abnormalities in the genome and transcriptome. One key feature is the involvement of chromosomal rearrangements creating fusion genes. Recent RNA-sequencing technology has uncovered that fusions which are not caused by chromosomal rearrangements, but rather meditated at transcription level, are common in both healthy and diseased cells. Such fusion transcripts have been proven highly associated with prostate cancer development and progression. To discover novel fusion transcripts, we analyzed RNA sequencing data from 44 primary prostate tumors and matched benign tissues from The Cancer Genome Atlas. Twenty-one high-confident candidates were significantly enriched in malignant vs. benign samples. Thirteen of the candidates have not previously been described in prostate cancer, and among them, five long intergenic non-coding RNAs are involved as fusion partners. Their expressions were validated in $\mathbf{5 0}$ additional prostate tumor samples and seven prostate cancer cell lines. For four fusion transcripts, we found a positive correlation between their expression and the expression of the $3^{\prime}$ partner gene. Among these, differential exon usage and qRT-PCR analyses in particular support that SLC45A3-ELK4 is mediated by an RNA polymerase read-through mechanism.

\section{INTRODUCTION}

Prostate cancer is world-wide the second most frequently diagnosed cancer type and the sixth leading cause of cancer-related death in men [1]. In the past decade, several discoveries of genetic alterations and gene expression abnormalities have revealed important molecular understanding to prostate cancer development and progression [2-5]. One predominant finding is the common expression of fusion genes in prostate cancer [5-7]. The most well-known is the fusion between TMPRSS2 and $E R G$, which are present in nearly half of the prostate cancers [5]. Also several other ETS transcription factors are recurrently overexpressed, where these are juxtaposed to strongly expressed genes [8-10]. The upstream fusion partners may or may not be regulated by androgens $[9,11]$. A number of common fusion transcripts generated from chromosomal rearrangements (e.g. translocation, insertion, deletion and inversion) have been detected by application of various high-throughput technologies [3, 4, 12-15].

Fusion transcripts, also known as chimeric RNAs, can also be generated without chromosomal rearrangements. For example, RNA polymerase read-through between two neighboring genes encoded on the same DNA strand and/ or trans-splicing of pre-mRNA (i.e. two separated premRNAs are spliced together and form a fusion transcript) are two mechanisms that give rise to fusion transcripts [16-18]. Unlike the classical fusion gene TMPRSS2-ERG, these fusion transcripts are the result of RNA processing and recombination without evidence of rearrangements on the DNA-level. Recent transcriptome-based analyses have revealed that transcription-induced gene fusions are commonly present in normal cells and tissues [14, 19, 20,46]. Many of them probably represent stochastic events with little or no impact on cellular functions, whereas others, such as SLC45A3-ELK4 and TMEM79-SGM5, have 
showed a high association with prostate cancer and indicate involvement in pathological processes [21, 22]. The use of next-generation sequencing technologies has advanced the identification of novel fusion transcripts in cancer samples, but it is still challenging to distinguish oncogenic driver fusions from passenger aberrations [13, 22-24]. The fact that fusion transcripts are present both in malignant and benign tissues provides an additional level of molecular complexity to consider in the search for biomolecules which are relevant to prostate cancer development, detection and treatment. Previous studies have only to a limited degree sought to capture the aberrant expression profile of fusion transcripts in cancer tissues $[17,22,25,26]$.

In this study, we aimed to identify recurrent and overexpressed fusion transcripts in prostate cancer. To achieve this, we analyzed the whole transcriptome sequencing data of 44 pairs of primary prostate tumors and adjacent normal tissues generated by The Cancer Genome Atlas (TCGA) [3]. Following the nomination of recurrent and overexpressed fusion transcripts, their expression patterns were further evaluated and validated using RNA sequencing data from 50 additional prostate tumor samples and seven prostate cancer cell lines. The inter-patient tumor-heterogeneity was explored by hierarchical clustering of the expression profiles of the fusion transcripts across the cohorts. Overall, our results revealed a complex landscape of novel and overexpressed fusion transcripts in prostate cancer. These transcripts probably represent a new repertoire for the discovery of novel cancer biomarkers and therapeutic targets.

\section{RESULTS}

\section{Fusion transcript discovery}

To identify fusion transcripts, we analyzed RNAseq data from TCGA prostate cancers by use of deFuse software. Initially, deFuse predicted 10,624 fusion transcripts across 44 paired tumor and benign prostate samples, and 1,218 candidates were considered further as they showed positive signals in at least five tumor samples (Figure 1). Of these, 175 fusion transcripts with both a significant enrichment of detected number ( $p<0.05$, Fisher's exact test; see Materials and Methods) and overexpression level in tumor vs. benign $(p<0.05$, Wilcoxon rank-test) were retained for further analysis. To control the false-positive identification of fusion partners due to sequence homology, 89 fusion transcripts with a large proportion $(>0.6)$ of multiple spanning reads to total spanning reads were removed. This reduced the candidates to 86. Furthermore, 60 fusion transcripts whose partner genes are related to overlapping and pseudo-genes were filtered out (Figure 1). Altogether, a set of 21 reliable fusion transcripts was finally nominated (Table 1). Analysis of the Illumina body map RNA-seq dataset (ArrayExpress accession ID E-MTAB-513 and European
Nucleotide Archive accession ID ERP000546) shows that none of the 21 fusion transcripts are detected from RNA-seq data of 12 human healthy tissues, which also included one sample of prostatic tissue. However, in the TCGA data, expressions of the 21 fusion transcripts were detected at various levels in 0 to 30 of the 44 benign tissue samples (Table 1). Hierarchical clustering of normalized expression values of the 21 fusion transcripts reveals that most of them have large variations in their expression profiles across the patient cohort, and no distinct subgroups were identified (Supplementary Figure 1). Such divergent expression signatures may be indicative of tumor heterogeneity at the transcriptomic level.

\section{Novel fusion transcripts with high prevalence in prostate cancers}

For the 21 identified fusion transcripts, the involved partner genes are mostly localized within $60 \mathrm{~kb}$ on the same chromosome, except TMPRSS2-ERG (intrachromosomal, distance approx. $3 \mathrm{Mb}$ ) and SSBP2-SPNE4 (interchro mosomal). Thirteen of the fusion transcripts have not previously been described in prostate cancer (Table 1). The remaining eight are known fusion transcripts (e.g. TMPRSS2-ERG, C9orf163-SEC16A, SMG5-TMEM79, and $K L K 4-K L K 3)[5,21,22]$, of which TMPRSS2-ERG is the most common, and was found to be positive in 19 (45\%) of the 44 primary tumor samples (Table 1 ).

Among the novel fusion transcripts, eight are between pairs of classical protein-coding genes, whereas five include long intergenic non-coding RNA (lincRNA) as one of the fusion partners (see Table 1). Fusion transcripts such as PXDN-AC144450.2 and ACER3-B3GNT6 have an overrepresentation in tumors and underrepresentation in benign tissues with highly significant $p$-values $(<1 \mathrm{E}-05)$. In comparison, the fusion transcripts $R P 11$ 17A19.1KCTD1, ZNF841-ZNF432, and ACSS1-APMAP show a common presence in normal samples as well, but they were found to be overexpressed in tumor $v s$. benign tissues ( $p<0.05$, Wilcoxon rank-test). To further confirm the high recurrence of these chimeras, an independent dataset of 50 additional primary tumors and seven prostate cancer cell lines were evaluated for the presence of any of the 13 novel fusion transcripts. We found that most of the transcripts could be found in similar proportions as in the 44 tumor-benign sample pairs (Supplementary Table 1). Only RP11_17A19.1-KCTD1 and RP11_321F6.1-SMAD6 show a slightly lower frequency from the 50 tumor samples. Moreover, four of the new nominated candidates were selected for RT-PCR validation, and three of them were successfully validated in five or all of the six prostate cancer cell lines (Supplementary Table 2). The breakpoints of fusion transcripts were verified by Sanger sequencing, and are identical to those found in RNA-seq data.

Next, we assessed whether the fusion transcripts can give rise to in-frame fusion proteins. In Table 1, we list 
Table 1: Nominated fusion transcripts

\begin{tabular}{|c|c|c|c|c|c|c|c|c|c|c|c|c|c|c|}
\hline Gene symbols & ChromA' & ChromB' & Strand $^{2}$ & $\begin{array}{c}\text { Distance between } \\
\text { fusion partners (bp) }\end{array}$ & $\begin{array}{c}\text { Fusion junction } \\
\text { at splice site }\end{array}$ & $\begin{array}{l}\text { Potential in- } \\
\text { frame protein }\end{array}$ & $\begin{array}{l}\text { No. of positive } \\
\text { tumor samples }\end{array}$ & $\begin{array}{l}\text { No. of positive } \\
\text { benign samples }\end{array}$ & Fisher_P ${ }^{3}$ & Exp_A $(T)^{4}$ & $\operatorname{Exp}_{-} V(T)^{5}$ & Exp_A $_{-}(\mathrm{B})^{4}$ & Exp_V $(B)^{5}$ & Wilcoxon_P ${ }^{o}$ \\
\hline TMPRSS2-ERG & 21 & 21 & -- & 2802774 & $\overline{\mathrm{Y}}$ & $\bar{N}$ & 19 & $\overline{0}$ & $1.6 \mathrm{E}-06$ & 6.20 & 5.36 & 0 & * & * \\
\hline$K L K 4-K L K 3$ & 19 & 19 &.+ & 45588 & $\mathrm{~N}$ & $\mathrm{~N}$ & 17 & 5 & $3.0 \mathrm{E}-03$ & 1.38 & 2.89 & 0.59 & $2.6 \mathrm{E}-02$ & $9.6 \mathrm{E}-03$ \\
\hline DNAJBI-TECR & 19 & 19 & -+ & 333 & $\mathrm{~N}$ & $\mathrm{~N}$ & 31 & 8 & $6.7 \mathrm{E}-07$ & 0.25 & $2.3 \mathrm{E}-02$ & 0.14 & $5.8 \mathrm{E}-03$ & $1.7 \mathrm{E}-02$ \\
\hline SMG5-TMEM79 & 1 & 1 & -+ & 110 & $\mathrm{~N}$ & $\mathrm{~N}$ & 20 & 6 & $1.0 \mathrm{E}-03$ & 0.53 & $1.2 \mathrm{E}-01$ & 0.29 & $4.6 \mathrm{E}-03$ & $1.6 \mathrm{E}-02$ \\
\hline GOLMI-NAA35 & 9 & 9 & -+ & 3848 & $\mathrm{~N}$ & $\mathrm{~N}$ & 42 & 30 & $8.2 \mathrm{E}-04$ & 0.49 & $1.1 \mathrm{E}-01$ & 0.29 & $2.5 \mathrm{E}-02$ & $5.8 \mathrm{E}-04$ \\
\hline SMG5-PAQRG & 1 & 1 & -- & 1134 & $\mathrm{~N}$ & $\mathrm{~N}$ & 11 & 1 & $1.7 \mathrm{E}-03$ & 0.21 & $2.4 \mathrm{E}-02$ & 0.15 & * & $*$ \\
\hline C9orf163-SECl6A & 9 & 9 & +- & 5806 & $\mathrm{~N}$ & $\mathrm{~N}$ & 34 & 11 & $8.2 \mathrm{E}-07$ & 0.56 & $1.2 \mathrm{E}-01$ & 0.27 & $2.3 \mathrm{E}-02$ & $2.1 \mathrm{E}-03$ \\
\hline$S L C 45 A 3-E L K 4$ & 1 & 1 & -- & 25889 & $\mathrm{Y}$ & $\mathrm{Y}$ & 14 & 5 & $1.8 \mathrm{E}-02$ & 0.37 & $1.9 \mathrm{E}-01$ & 0.20 & $4.3 \mathrm{E}-03$ & $2.2 \mathrm{E}-02$ \\
\hline \multicolumn{15}{|l|}{ Novel fusion transcripts } \\
\hline PXDN-ACl44450.2 & 2 & 2 & -- & 6468 & $\mathrm{~N}$ & $\mathrm{~N}$ & 27 & 1 & $4.2 \mathrm{E}-10$ & 0.47 & 7.3E-01 & 0.14 & * & * \\
\hline ACER3-B3GNT6 & 11 & 11 & + & 7544 & $\mathrm{~N}$ & $\mathrm{~N}$ & 19 & 1 & $2.1 \mathrm{E}-06$ & 0.49 & $1.9 \mathrm{E}-01$ & 0.13 & * & * \\
\hline DSCCI-KB_1471A8.I & 8 & 8 & -+ & 11409 & $\mathrm{~N}$ & $\mathrm{~N}$ & 6 & 0 & $1.3 \mathrm{E}-02$ & 0.07 & $6.5 \mathrm{E}-04$ & 0 & * & * \\
\hline ACSSI-APMAP & 20 & 20 & -- & 13253 & $\mathrm{~N}$ & $\mathrm{Y}$ & 29 & 20 & $4.3 \mathrm{E}-02$ & 0.31 & $3.1 \mathrm{E}-02$ & 0.20 & $1.1 \mathrm{E}-02$ & $4.6 \mathrm{E}-02$ \\
\hline SPON2-CTBPI & 4 & 4 & -- & 2486 & $\mathrm{~N}$ & $\mathrm{~N}$ & 14 & 2 & $8.2 \mathrm{E}-04$ & 0.53 & $1.6 \mathrm{E}-01$ & 0.12 & $1.9 \mathrm{E}-03$ & * \\
\hline SSBP2-CPNE4 & 5 & 3 & NA & NA & $\mathrm{N}$ & $\mathrm{N}$ & 16 & 1 & $3.1 \mathrm{E}-05$ & 1.42 & 4.39 & 1.08 & * & * \\
\hline NSUNA-FAAH & 1 & 1 & + & 29113 & $\mathrm{~N}$ & $\mathrm{Y}$ & 6 & 0 & $1.3 \mathrm{E}-02$ & 0.31 & 3.4E- -02 & 0 & * & * \\
\hline TMEM219-TAOK2 & 16 & 16 & ++ & 589 & $\mathrm{Y}$ & $\mathrm{Y}$ & 10 & 2 & $2.5 \mathrm{E}-02$ & 0.11 & 4.1E- 03 & 0.06 & $9.5 \mathrm{E}-04$ & * \\
\hline RPII_32IF6.I-SMAD6 & 15 & 15 & ++ & 16434 & Y & $\mathrm{N}$ & 5 & 0 & $2.8 \mathrm{E}-02$ & 0.18 & $2.4 \mathrm{E}-02$ & 0 & * & * \\
\hline ZNF841-ZNF432 & 21 & 21 & -- & 15613 & $\mathrm{~N}$ & $\mathrm{Y}$ & 15 & 5 & $1.0 \mathrm{E}-02$ & 0.15 & 4.8E- 03 & 0.08 & $1.0 \mathrm{E}-03$ & $1.6 \mathrm{E}-02$ \\
\hline ZNF551-ZNF776 & 19 & 19 & ++ & 56997 & $\mathrm{~N}$ & $\mathrm{Y}$ & 9 & 1 & 7.4E-03 & 0.11 & $3.0 \mathrm{E}-03$ & 0.07 & * & * \\
\hline RPII_I7A19.I-KCTDI & 18 & 18 & -- & 30220 & Y & $\mathrm{N}$ & 25 & 6 & $2.0 \mathrm{E}-05$ & 0.58 & $3.2 \mathrm{E}-01$ & 0.18 & $1.3 \mathrm{E}-02$ & $7.2 \mathrm{E}-03$ \\
\hline $\begin{array}{l}\text { FAM } 833 \text { - } \\
\text { RPII } 429.117 .6\end{array}$ & 8 & 8 & -+ & 339 & $\mathrm{~N}$ & $\mathrm{~N}$ & 15 & 0 & $1.4 \mathrm{E}-04$ & 0.22 & $2.3 \mathrm{E}-02$ & 0 & * & * \\
\hline
\end{tabular}

Chromosome numbers for the $\mathrm{A}$ and $\mathrm{B}$ genes, i.e. upstream and downstream fusion partners

${ }^{3} P$ value of Fisher's exact test for difference in positive fraction among tumor $v s$. benign samples.

The average expression value for fusion transcripts in tumor and benign samples.

$P$ value of Wilcoxon rank test for differential expression between tumor and benign samples.

*Too small sample size of benign tissues for statistics test.

NA: Fusion partners are located on different chromosomes.

that five of the novel fusion transcripts may translate into fusion proteins with truncated C-terminal of the header gene and an in-frame translation of the tail gene. One example, NSUN4-FAAH, may translate into a putative fusion protein composed of the Methyltransf_26 domain of NSUN4 (exons 1 to 6) and the Amidase domain of
FAAH (exons 2 to 15; Supplementary Figure 2). Two in-frame fusion transcripts involved partner genes encoding zinc finger family proteins, ZNF841-ZNF432 and ZNF551-ZNF776, which might lead to coupling of two ZNF domains. Furthermore, the fusion transcript TMEM219-TAOK2, has intact splicing sites and reading

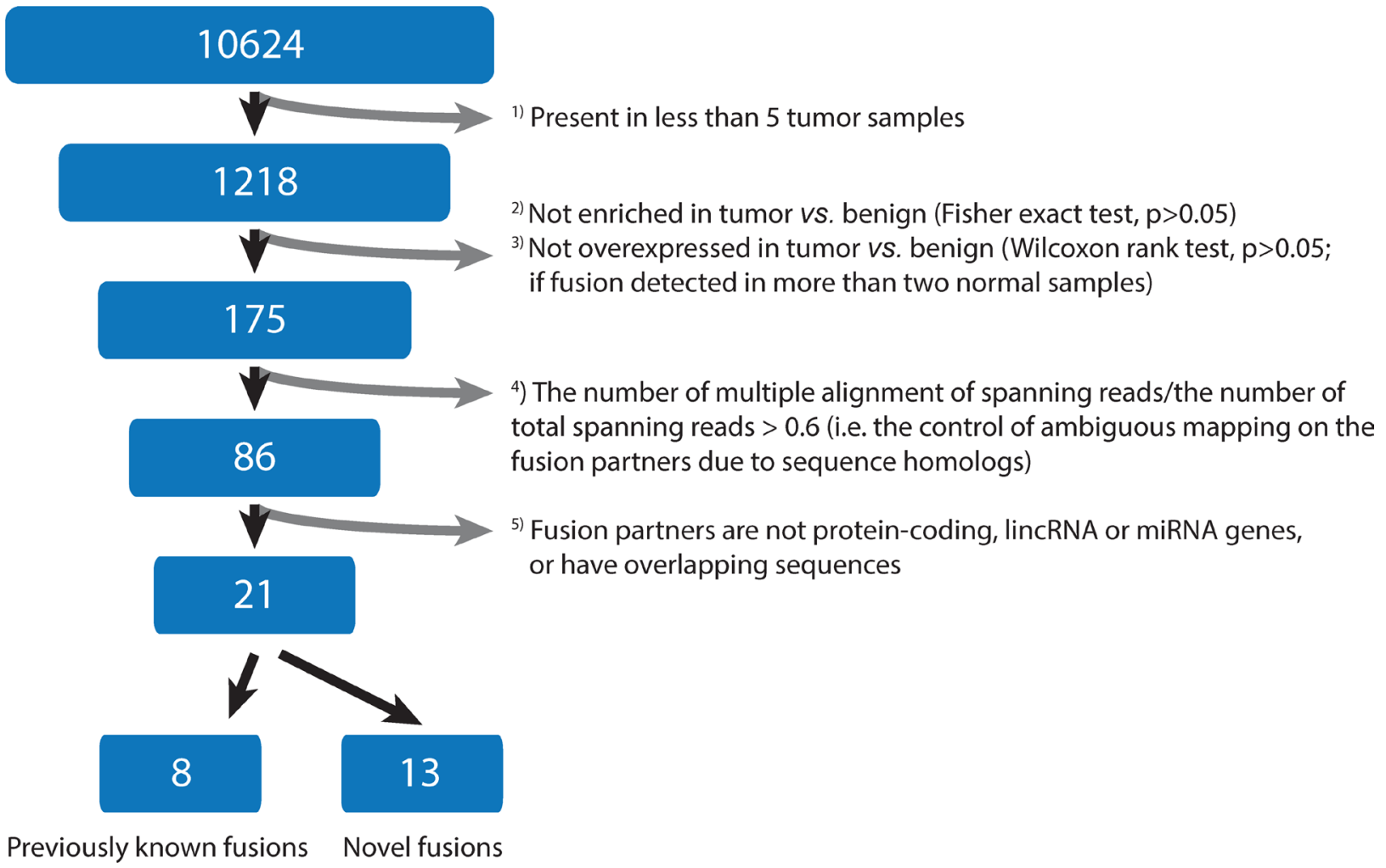

Figure 1: Flowchart of fusion transcript filtering. The identified fusion transcripts were filtered in a successive manner, following the initial analysis of 44 paired tumor and benign prostate samples by deFuse software. 
frames of both fusion partners. The fusion protein may include parts encoded from the first five exons (240 amino acids) of TMEM219 and exons 2 to 16 (1122 C-terminal amino acid residues) of $T A O K 2$, which combines a transmembrane and a serine/threonine kinase (S_TKc) domain (see Figure 2).

In addition, we found that two fusion transcripts, RP11_17A19.1-KCTD1 and RP11_321F6.1-SMAD6, are predicted not to encode fusion proteins. Instead, both combine a lincRNA gene as the upstream partner and a protein-coding gene as the downstream partner, using intact exon splicing sites from their fusion partner genes. We identified on average 20 and 5 split reads supporting the junctions (exon1-exon2 (RP11_17A19.1$K C T D 1$; Supplementary Figure 3) and exon3-exon2 (RP11_321F6.1-SMAD6; Supplementary Figure 4), respectively). The 3' gene partner retains an intact $(K C T D 1)$ or truncated (SMAD6) coding sequence in the putative fusion proteins. In particular, the fusion transcript RP11_17A19.1-KCTD1 is highly prevalent, being detected in 25 of the $44(56 \%)$ and 14 of the $50(28 \%)$ prostate tumors. This fusion transcript was as well detectable in six of the 44 benign prostate samples. Importantly, its expression level in tumors is significantly higher than that in benign prostates ( $p=7.2 \mathrm{E}-03$, Wilcoxon rank-test; Table 1).

\section{Expression of fusion transcripts and their $3^{\prime}$ fusion partner genes}

We compared the expression levels of fusion transcripts to these of the fusion partner genes, in both tumor and benign prostate samples. Our results show that most fusion partner genes are expressed at a moderate or low level. Of interest, several of the $3^{\prime}$ fusion partners are differentially expressed between samples with and without detection of the fusion transcripts. For instance, B3GNT6 is highly expressed in the ACER3-B3GNT6 positive tumor samples, and almost absent in both the fusionnegative tumor samples ( $p=9.9 \mathrm{E}-12)$ and benign samples ( $p=2.1 \mathrm{E}-13$, Wilcoxon rank-test; Figure 3A). Similarly, the fusion partner ELK4 is up-regulated in tumor samples expressing the $S L C 45 A 3-E L K 4$ fusion in comparison with fusion-negative tumor samples $(p=0.01)$ and benign samples ( $p=6.3 \mathrm{E}-05$, Wilcoxon rank-test; Figure $3 \mathrm{~B}$ ). The same pattern was found from the 50 additional tumor samples (Figure 3C and 3D). Moreover, the expression of fusion transcripts ACER3-B3GNT6 and SLC45A3-ELK4 were found positively correlated to the expression of their downstream partners ( $p=2 \mathrm{E}-03$ and $p=3 \mathrm{E}-04$, Spearman correlation test; Figure $3 \mathrm{E}$ and $3 \mathrm{~F}$ ). In differential exonlevel expression analysis, exon 1 (only in wild type) of ELK4 shows a higher expression in fusion negative tumors than in fusion positive tumors (Supplementary Figure 7A). In comparison, exons 2-6 (sum of fusion and wild type) of ELK4 are stronger expressed in fusion positive tumors
(Supplementary Figure 7A). Quantitative RT-PCR analyses confirm that the expression of ELK4 exon 2 (sum of fusion and wildtype) is significantly higher than that of exon 1 (wildtype) in 39 prostate tumor samples ( $p=3.1 \mathrm{E}-10$, Wilcoxon rank-test; Supplementary Figure 8A). And the expression of the fusion transcript shows a significant positive correlation to that of ELK4 exon 2 ( $r=0.83$ and $p=5.6 \mathrm{E}-11$, Spearman correlation test; Supplementary Figure $8 \mathrm{~B})$. There was no correlation between the expression of ELK4 exon 2 and ELK4 exon $1(r=-0.1$ and $p=0.43$, Spearman correlation test; Supplementary Figure 8C). These results support that the induced expression of the downstream fusion partner is driven by the expression of the fusion transcript, which again most likely is mediated by RNA polymerase read-through from the upstream partner gene. It further indicates that the formation of transcription-induced fusion transcripts may be accompanied with induced expression of the downstream fusion partner.

\section{DISCUSSION}

In this study, we carried out a comprehensive survey of fusion transcripts in RNA-sequencing data from 44 pairs of prostate cancer and benign tissues being sequenced by the TCGA consortium [3]. Thirteen novel recurrent fusion transcripts were nominated using stringent filtering criteria. In general, these chimeras are characterized by having partner genes which are localized less than $60 \mathrm{~kb}$ apart and are transcribed from the same strand (10/13 gene pairs). Thus, they are likely produced by transcription-induced fusion events, and not genomic rearrangements. As several of the fusion transcripts were also present in the benign prostate samples (Table $1)$, the selection of sufficient normal samples as control is therefore of key importance to distinguish potential driver fusions from passengers. If the fusion transcripts are only detected in 1-2 normal samples (like $A C E R 3-$ $B 3 G N T 6$ and $P X D N-A C 144450.2)$, we suggest that they are probably involved in the cancer development. Their rare presence in benign prostate tissues may represent a "field effect" within the normal epithelium preceding histological change [27], or a possible contamination by marginal tumor cells in the normal tissue during sampling. Thus, a further validation from more patient samples and cell lines would be helpful to clarify the feature of such fusion event in normal tissue. On the other hand, several fusion transcripts (e.g. SLC45A3-ELK4 and C9orf163$S E C 16 A$ ) show a common presence in normal samples (Table 1). The comparison of the expression level of fusion transcripts in tumors $v s$. benign tissues enabled us to nominate the ones overexpressed in cancers as likely involved in cancer-related pathological processes. Regardless of the fusion transcripts being detected in benign tissues, their high prevalence in the 94 investigated tumor samples indicate that the expression of these fusion 

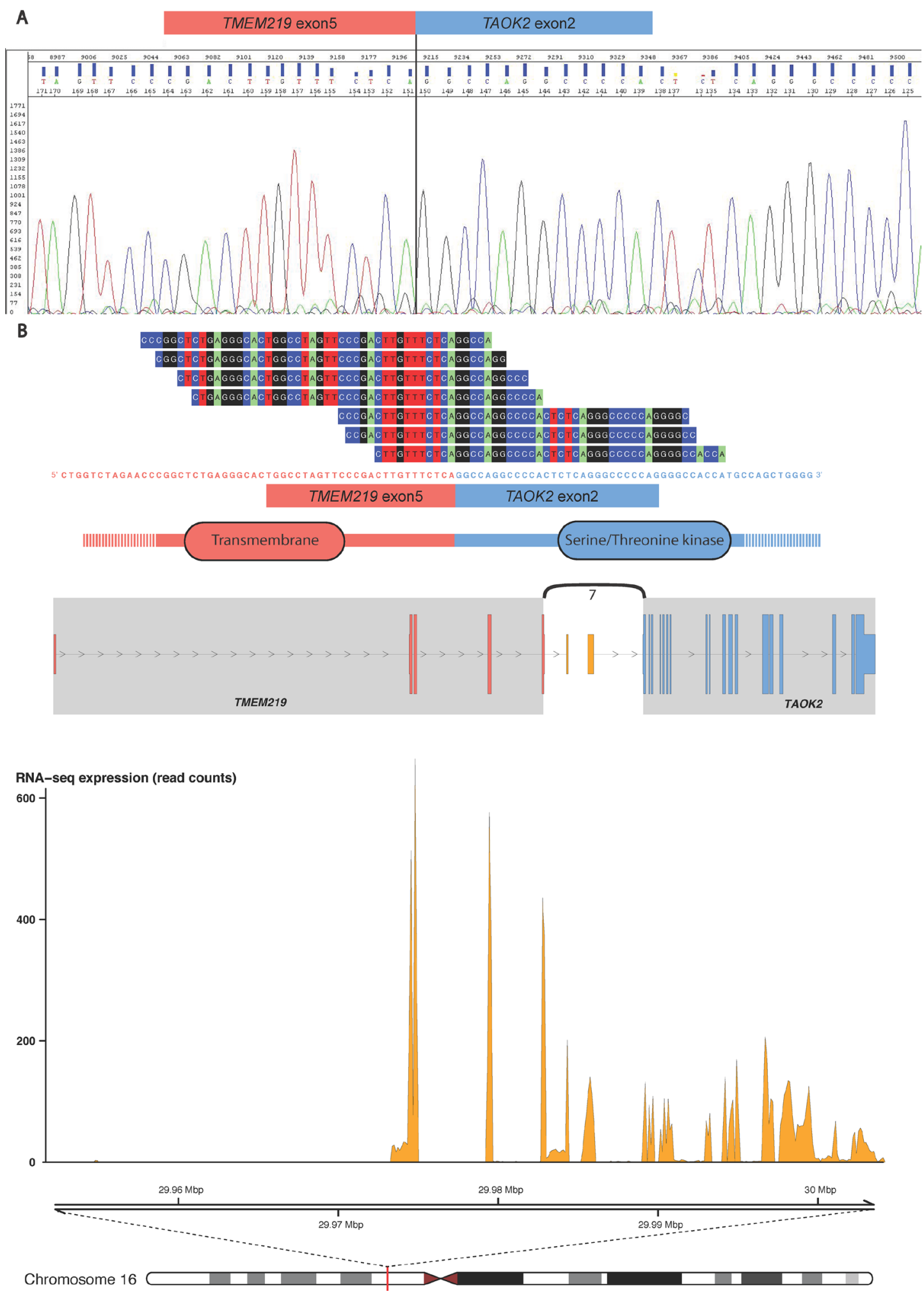

Figure 2: The TMEM219-TAOK2 fusion transcript. (A) The fusion transcript was detected from the VCaP, DU145, PC3, 22Rv1, NCI-H660 and LNCaP cell lines (see Supplementary Table 2), and the specific breakpoint was verified by Sanger sequencing. (B) Example data from the TCGA prostate tumor "TCGA-HC-7211-01A-11R-2118-07" shows seven split reads spanning the chimeric transcript breakpoint, from exon 5 of TMEM219 (ENST00000414689) to exon 2 of TAOK2 (ENST00000279394). The fusion transcript is predicted to include an in-frame open reading frame encoding a chimeric protein with the combination of transmembrane and serine/threonine kinase domains. The genomic view of the fusion event is from the top showing annotated exons of the fusion partner genes and the number of split reads supporting breakpoint (curved line), the RNA expression levels (read counts), and genomic coordinates for the fusion transcript in mega base pairs from the p-telomere of chromosome 16. 
transcripts may be relevant to pathological processes and malignant transformation. Our study expands the scope of classical fusion genes derived by chromosome rearrangement to include more potential driver fusions mediated at the transcriptome level.

Two important mechanisms have been proposed to mediate the formation of transcription-induced fusion genes, RNA polymerase read-through and trans-splicing (see review [28]). Although both can produce chimeras

\section{A B3GNT6 expression}

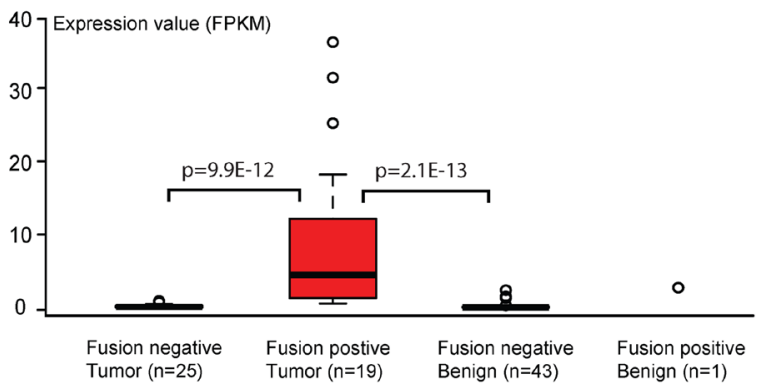

\section{B3GNT6 expression}

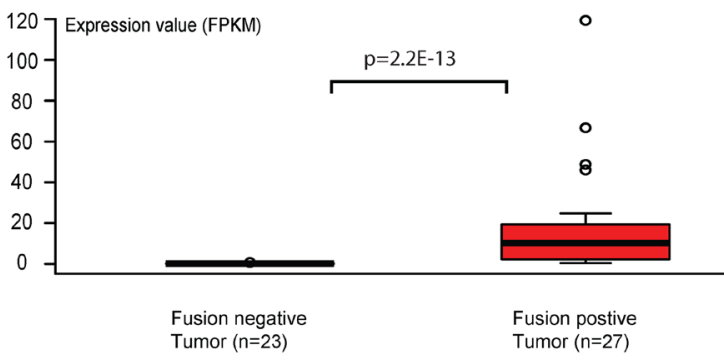

$E$

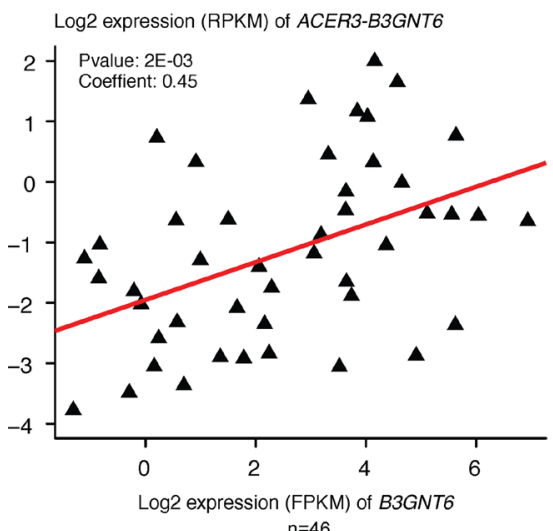

by splicing events, the read-through scenario may be coupled with an induced expression level of the $3^{\prime}$ gene partner, since the fusion transcript is transcribed from the promoter of the $5^{\prime}$ gene. In this study, up-regulation of $3^{\prime}$ gene partners were seen for the fusion transcripts $S L C 45 A 3$ ELK4, ACER3-B3GNT6, ZNF841-ZNF432 and PXDN$A C 144450.2$, when comparing fusion-positive to fusionnegative tumor samples (Figure 3 and Supplementary Figure 6). A significant differential usage of exon was

B ELK4 expression
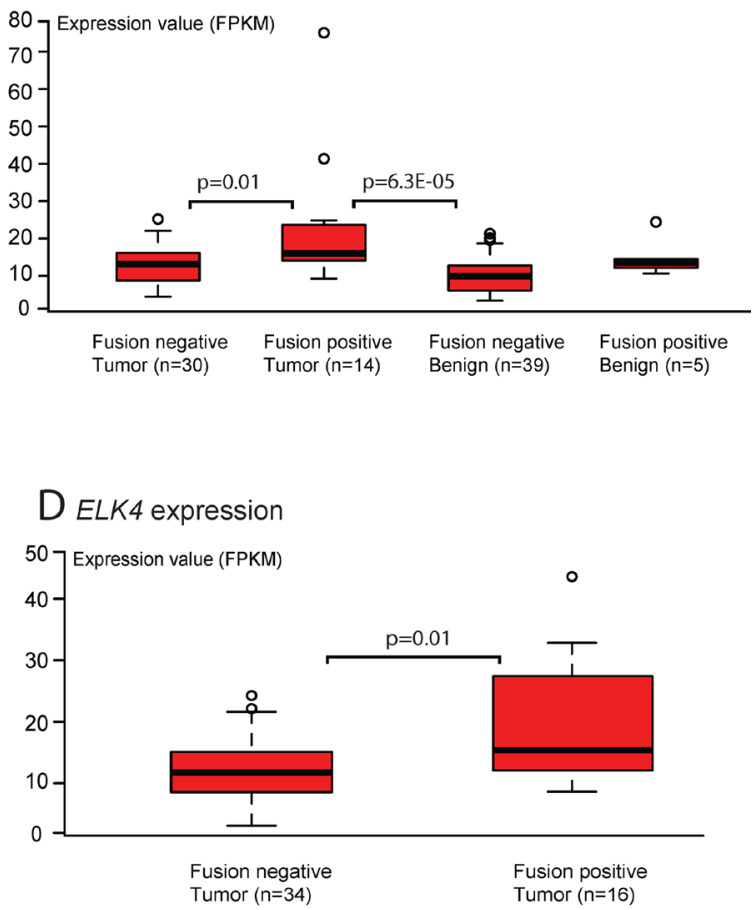

$\mathrm{F}$

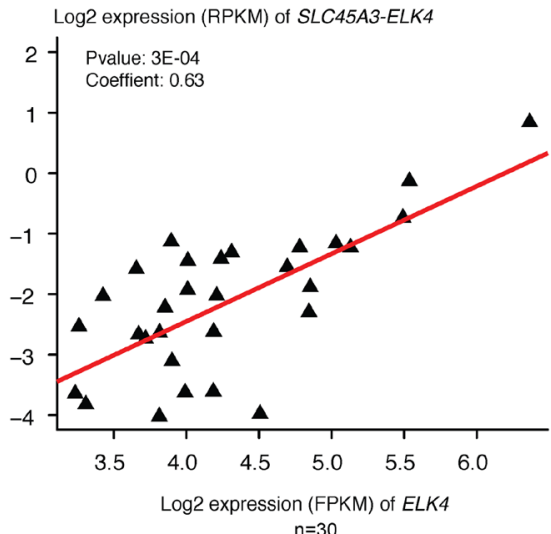

$n=30$

Figure 3: Differential expression of $\mathbf{3}^{\prime}$ fusion partner genes. (A) and (B) show the expression of the $3^{\prime}$ partner genes, $B 3 G N T 6$ and ELK4, of the fusions ACER3-B3GNT6 and SLC45A3-ELK4 in 44 pairs of tumor and benign prostate samples. (C) and (D) show the expression of $3^{\prime}$ partner genes B3GNT6 and ELK4 in 50 additional prostate tumors. (E) and (F) show the correlation between the expression of ACER3-B3GNT6 and SLC45A3-ELK4 and their respective 3' partner genes, B3GNT6 and ELK4. X and Y axes represent $\log 2$ transformed expression values (FPKM, fragments per kilobase of transcript per million mapped reads; RPKM, reads per kilobase of transcript per million mapped reads). 
shown in $3^{\prime}$ partner gene ELK4, and less so for the $3^{\prime}$ partner gene ZNF432 (Supplementary Figure 7A and 7B). Quantitative RT-PCR analyses of exons up- and downstream of the breakpoint in the fusion partner ELK4 (exon1 and 2), support that the expression of fusion transcript contributes significantly to the up-regulation of the $3^{\prime}$ gene partner ELK4 (Supplementary Figure 8). For the 3' fusion partners $B 3 G N T 6$ and $A C 144450.2$ this was not seen (Supplementary Figure 7C and S7D), and we cannot rule out other mechanisms than RNA polymerase read-through.

In addition, B3GNT6 was also found as $3^{\prime}$ gene partner in the fusion SOD2-B3GNT6 (data not shown), this fusion transcript was only detected in one tumor sample. Its low prevalence suggests that the increased expression of B3GNT6 is mainly mediated by the control of the $A C E R 3$ promoter. In addition to the $S L C 45 A 3-E L K 4$, we found three other fusion transcripts involving $E L K 4$ (data not shown). However, in all these, ELK4 was involved as the $5^{\prime}$ partner gene. Overall, our findings indicate that each of the four fusions is transcribed as a single transcript, generated by RNA polymerase read-through of both partner genes, and their presences contribute to a higher expression of the 3' partner genes. However, the formation of fusion transcripts by a read-through mechanism is only one possible explanation for the increased expression of $3^{\prime}$ partner genes. Since the transcription of parental genes is not broken off by the read-through, also other factors may contribute to the up-regulation of partner genes.

Despite the high recurrence of 13 novel fusion transcripts in prostate tumors, their biological roles are not obvious. As shown in Supplementary Table 3, fusion formation may have impact on several cellular pathways, such as the apoptotic process (TMEM219-TAOK2), fatty acid metabolism (NSUN4-FAAH), glycoprotein biosynthesis (ACER3-B3GNT6), membrane trafficking (SSBP2-CPNE4), cell adhesion (SPON2-CTBP1), and transcript regulation (ZNF841-ZNF432 and ZNF551-ZNF776). The fusion transcripts can be translated into in-frame fusion proteins with intact or truncated coding region of the fusion partner genes. They may as well have new $3^{\prime}$-untranslated regions, which could alter the translational efficiency.

Three fusion transcripts, TMEM219-TAOK2, NSUN4-FAAH, and ACER3-B3GNT6, show a functional implication to prostate cancer development and progression. Firstly, TMEM219 is a transmembrane protein located in the plasma membrane, whereas TAOK2 (also named "prostate-derived sterile 20-like kinase 1") is a serine/ threonine protein kinase that is usually expressed in the nucleus. Both proteins are involved in the apoptotic process and are related to development of prostate cancer [29, 30], in particular TAOK2 is critical in activation of MAPKK pathway and regulation of cell growth [31]. The putative chimeric protein combines an intact transmembrane domain and a serine/threonine kinase domain (Figure 2), and may induce the kinase activity outside of the nucleus. For the fusion NSUN4-FAAH (Supplementary Figure 2),
NSUN4 is an rRNA methyltransferase involved in mitochondrial ribosome assembly and FAAH is a fatty acid amide hydrolase that participates in fatty acid catabolic process $[32,33]$. The putative NSUN4-FAAH chimeric protein shows a domain rearrangement which integrates the core catalytic function of the two partners. Although no studies have indicated a possible role in tumorigenesis for the two genes, FAAH has previously been reported to be highly expressed in prostate tissue [34, 35]. As discussed, the presence of fusion ACER3-B3GNT6 (Figure 3 and Supplementary Figure 5) is significantly associated with an up-regulation of B3GNT6 expression level. B3GNT6 is short for $\beta-1,3-\mathrm{N}$-acetylglucosaminy transferase 6 and has an important role in the synthesis of mucin-type O-glycans in the Golgi apparatus. Increased B3GNT6 expression has previously been reported in prostate cancer, and it may be involved in the metastatic capacity of cancer cells [36]. In this study, a high expression of B3GNT6 was found in 19 of $44(43 \%)$ tumor samples.

We found that lincRNAs are commonly involved in the formation of fusion transcripts. As shown in Table 1, five of the 13 nominated fusion candidates included lincRNAs (two as upstream partner and three as downstream partner). One previously characterized lincRNA ( RP11_321F6.1, also known as $P C A T 18$ ), is shown to be a regulator of prostate cancer cell proliferation, invasion and migration [37]. Another fusion transcript frequently expressed in prostate tumors involves the lincRNA AC144450.2 with unknown function. Since lincRNAs regulate gene expression in cancer cells and may play oncogenic or tumor suppressive roles [38], the investigation of biological implication of lincRNA fusion transcripts may provide new insights into tumorigenesis at the transcription regulatory level, and can be a source of cancer biomarker and therapeutic targets.

Notably, no partner genes of the novel fusion transcripts belong to the ETS transcription factor gene family. Further, most of the novel fusions are expressed in less than $50 \%$ of the tumor samples and show a scattered distribution across the cohorts (Supplementary Figure 1). This supports a strong inter-tumor heterogeneity, which is also clearly indicated in earlier studies of DNA copy numbers, somatic mutations and epigenetic alterations $[2,3,24,39]$. In conclusion, we have here identified a set of novel fusion transcripts being overexpressed in prostate cancer. These fusion transcripts form an additional layer of cellular complexity in prostate cancers with yet unknown implications for the development and management of this disease.

\section{MATERIALS AND METHODS}

\section{Data preparation}

Raw RNA sequencing data in fastq format from TCGA (44 pairs primary tumors and benign tissues; 50 unpaired primary tumors) were downloaded from 
Cancer Genome Hub (CGHub, https://cghub.uscs.edu, now changed to GDC data portal: https:/gdc-portal. nci.nih.gov). RNA sequencing data in BAM format for seven prostate cancer cell lines (Cancer Cell Line Encyclopedia) were obtained from CGHub repository and aligned reads were extracted using bam 2 fastq. The summary of RNA-seq data from the TCGA and Cancer Cell Line Encyclopedia is listed in Supplementary Table 4. Clinical data, i.e. pre- and post-operation values for prostate specific antigen (PSA) and Gleason scores, were downloaded from the FTP server of TCGA.

\section{Fusion detection and filtering}

We used the deFuse software (version 0.6.1), with hg19 human reference genome sequence and Ensembl release 69 annotation database, to predict fusion transcripts from the RNA sequencing data for both primary tumors and benign tissues [40]. Briefly, deFuse detected fusion transcripts through the identification of discordant read pairs and junction split reads. The preliminary deFuse output of fusion RNA predictions were generated according to the following criteria: 1) at least five discordant read pairs, 2) at least one junction split read and 3 ) deFuse classifier probability no less than 0.05 . To better evaluate the fusions that showed presence in both tumor and benign samples, we approximately quantified the expression of fusion transcripts by calculating the number of split reads mapped to breakpoint region (i.e. reads per kilobase of transcript per million mapped reads; measured as RPKM value).

Several specific filtering steps were further applied for reducing the number of false positives based on statistics data mining: 1) fusions had to be present in at least five tumor samples; 2) the number of fusions was enriched in tumors compared to that in benign prostates $(p<0.05$; Fisher's exact test); 3$)$ if fusions present in more than two benign prostates, they were retained only when the expression level was higher in tumor as compared to normal ( $p<0.05$; Wilcoxon signed rank test); 4) the ratio of multi-mapping spanning reads to total spanning reads should be less than 0.6 (i.e. the control of possible ambiguous mapping on both sides of the predicted breakpoints); 5) both gene partners of fusion transcripts had to be either protein-coding, lincRNA or miRNA genes, and putative fusions between overlapping genes were discarded. All fusion candidates nominated by these procedures were manually reviewed in the UCSC Genome Browser by BLAT (http://genome.ucsc.edu/cgibin/hgBlat?command=start) to double-check the sequence specificity in breakpoint region.

\section{Functional annotation}

Annotations of the gene partners involved in the formation of fusion candidates were assigned by Gene ontology (GO) terms in three major categories: Molecular function (MF), biological process (BP) and cellular component (CC) (http://www.geneontology.org/). The terms with evidence inferred from electronic annotation (IEA) were discarded in this study. Putative chimeric proteins that hold in-frame coding sequences were searched for structural domains and motifs against Pfam and SMART databases [41, 42]. A domain was considered present in the chimeric protein if it had an $e$-value $<=1$ E-6. Particular attention was paid to breakpoints that occurred outside of structural domains.

\section{Expression profile analysis}

RNA-seq raw reads were mapped onto the hg19 genome and exon-exon junction by splice-aware aligner TopHat 2.0.11 [43]. Gene expression profile for each sample was calculated using Cufflinks 2.2.0 based on the Ensembl gene annotation (release 69) [44], and the expression were quantified in fragments per kilobase of gene per million mapped reads (FPKM). The differential expression level of fusion transcripts $(i)$ between 44 pair tumor-benign samples

(j) was normalized using: value $_{i j}^{\prime}=\left(\frac{\operatorname{Texp}_{i j}-N \operatorname{Nexp}_{i j}}{\max _{j=1.44}\left|\operatorname{Texp}_{i j}-N \exp _{i j}\right|}\right)$, where value $_{\mathrm{ij}}{ }^{\prime}$ is the normalized differential expression value, $\operatorname{Texp}_{\mathrm{ij}}$ and $N \exp _{\mathrm{ij}}$ represent the expression levels of fusion transcript $i$ in tumor and matched normal tissue $j$. Hierarchical clustering analysis using Pearson correlation as distance matrix was done in R 3.1.2. Fusion breakpoints, the transcript annotation of gene partners and sequencing coverage were visualized using $\mathrm{R}$ package chimeraviz (submission to Bioconductor; https://www.bioconductor. org/packages/devel/bioc/html/chimeraviz.html). Differential exon usage analyses were performed by DEXSeq package after counting reads per exon using "dexseq_count.py" script [45]. Samples were normalized using function "estimateSizeFactor" and variability was estimated using function "estimateDispersons". Statistical testing of differential exon usage between fusion positive and negative conditions was done using function "testForDEU" ( $q$-value $<0.1$ was considered as significant).

\section{RT-PCR and validation of fusion transcripts on Prostate cancer cell lines}

RNA was isolated from six prostate cancer cell lines (DU145, PC3, VCaP, 22Rv1, NCI-H660 and LNCaP) using AllPrep DNA/RNA/miRNA Universal kit (Qiagen, Hilden, Germany), and cDNA synthesis was performed using the High Capacity cDNA Reverse Transcription kit (Applied Biosystems by ThermoFisher Scientific (AB), MA, USA). Validation was performed by RT-PCR across the predicted breakpoint regions. Reactions creating visible bands on an agarose gel were sequenced directly (AB 3730 DNA Analyzer, AB) to validate further the presence of the fusion transcripts (primers can be found in Supplementary Table 5; Supplementary Figure 9). 


\section{Quantitative real-time reverse transcriptase PCR}

Included in the analyses were RNAs from 39 fresh frozen prostate cancer samples from prostatectomies undertaken at the Oslo University Hospital-Radiumhospitalet during 2010-2012. The RNA was isolated and cDNA synthesized as described above for the cell lines. Expressions of the fusion transcript SLC45A3-ELK4 and of exons up- and downstream of the breakpoint in ELK4 (exon 1 and exon 2) were analyzed using three TaqMan assays (AB). The assays measuring the individual exons in ELK4 were as designed by $\mathrm{AB}$ (exon 1, Hs00360812; exon 2, Hs00360813_m1). The assay specifically measuring the chimeric fusion breakpoint (primers and probe in Supplementary Table 5) was custom designed with Primer Express 3.0 based on parameters given by the Primer Express software (AB). For each reaction, $10 \mathrm{ng}$ cDNA was used. For the predesigned assays, $5 \mu 1$ TaqMan Universal Master Mix II with UNG (2X), $0.5 \mu 1$ assay and $1.5 \mu 1 \mathrm{RNase}$ free water were added to a total volume per well of $10 \mu \mathrm{l}$. For the custom designed assay $0.09 \mu \mathrm{l}$ of each primer (100 uM), $0.2 \mu \mathrm{l}$ probe $(10 \mathrm{uM})$ and $1.62 \mu \mathrm{l}$ RNase free water were combined to a total volume of $10 \mu 1$. The reactions were incubated at $50^{\circ} \mathrm{C}$ for 2 minutes followed by $95^{\circ} \mathrm{C}$ for 10 minutes and 40 cycles of $95^{\circ} \mathrm{C}$ for 15 seconds 60 for 1 minute. Fluorescence was measured on an ABI 7900HT Fast Real-time PCR System (AB). All assays were run in triplicates on ABI 7900HT Fast Realtime PCR System, and expression levels were reported as the median cycle threshold $\left(\mathrm{C}_{\mathrm{T}}\right)$ of the triplicates.

\section{ACKNOWLEDGMENTS}

We are grateful to Mette Eknæs for help with cell culturing. We thank Torfinn Nome for discussion about fusion gene visualization. The study was funded by grants from the Norwegian Cancer Society (SZ was financed as a postdoc from the PR-2007-0166 grant), the South-Eastern Norway Regional Health Authority (ML was financed as a postdoc from the 2013107 grant), NorStore (for storage of computer files; project NS9013K), Notur (CPU hours from Abel supercomputer; project NN9313K), and the Research Council of Norway through its Centers of Excellence funding scheme (project number 179571).

\section{CONFLICTS OF INTEREST}

None.

\section{REFERENCES}

1. Jemal A, Bray F, Center MM, Ferlay J, Ward E, Forman D. Global cancer statistics. CA Cancer J Clin. 2011; 61:69-90.
2. Taylor BS, Schultz N, Hieronymus H, Gopalan A, Xiao Y, Carver BS, Arora VK, Kaushik P, Cerami E, Reva B, Antipin Y, Mitsiades N, Landers $\mathrm{T}$, et al. Integrative genomic profiling of human prostate cancer. Cancer cell. 2010; 18:11-22.

3. Cancer Genome Atlas Research Network. Electronic address scmo and Cancer Genome Atlas Research N. The Molecular Taxonomy of Primary Prostate Cancer. Cell. 2015; 163:1011-1025.

4. Baca SC, Prandi D, Lawrence MS, Mosquera JM, Romanel A, Drier Y, Park K, Kitabayashi N, MacDonald TY, Ghandi M, Van Allen E, Kryukov GV, Sboner A, et al. Punctuated evolution of prostate cancer genomes. Cell. 2013; 153:666-677.

5. Tomlins SA, Rhodes DR, Perner S, Dhanasekaran SM, Mehra R, Sun XW, Varambally S, Cao X, Tchinda J, Kuefer R, Lee C, Montie JE, Shah RB, et al. Recurrent fusion of TMPRSS2 and ETS transcription factor genes in prostate cancer. Science. 2005; 310:644-648.

6. Tomlins SA, Laxman B, Dhanasekaran SM, Helgeson BE, Cao X, Morris DS, Menon A, Jing X, Cao Q, Han B, Yu J, Wang L, Montie JE, et al. Distinct classes of chromosomal rearrangements create oncogenic ETS gene fusions in prostate cancer. Nature. 2007; 448:595-599.

7. Kumar-Sinha C, Tomlins SA, Chinnaiyan AM. Recurrent gene fusions in prostate cancer. Nat Rev Cancer. 2008; $8: 497-511$.

8. Tomlins SA, Mehra R, Rhodes DR, Smith LR, Roulston D, Helgeson BE, Cao X, Wei JT, Rubin MA, Shah RB, Chinnaiyan AM. TMPRSS2:ETV4 gene fusions define a third molecular subtype of prostate cancer. Cancer Res. 2006; 66:3396-3400.

9. Helgeson BE, Tomlins SA, Shah N, Laxman B, Cao Q, Prensner JR, Cao X, Singla N, Montie JE, Varambally S, Mehra R, Chinnaiyan AM. Characterization of TMPRSS2:ETV5 and SLC45A3:ETV5 gene fusions in prostate cancer. Cancer Res. 2008; 68:73-80.

10. Paulo P, Barros-Silva JD, Ribeiro FR, Ramalho-Carvalho J, Jeronimo C, Henrique R, Lind GE, Skotheim RI, Lothe RA, Teixeira MR. FLI1 is a novel ETS transcription factor involved in gene fusions in prostate cancer. Genes Chromosomes Cancer. 2012; 51:240-249.

11. Carver BS, Tran J, Chen Z, Carracedo-Perez A, Alimonti A, Nardella C, Gopalan A, Scardino PT, Cordon-Cardo C, Gerald W, Pandolfi PP. ETS rearrangements and prostate cancer initiation. Nature. 2009; 457:E1; discussion E2-3.

12. Maher CA, Kumar-Sinha C, Cao X, Kalyana-Sundaram S, Han B, Jing X, Sam L, Barrette T, Palanisamy N, Chinnaiyan AM. Transcriptome sequencing to detect gene fusions in cancer. Nature. 2009; 458:97-101.

13. Maher CA, Palanisamy N, Brenner JC, Cao X, KalyanaSundaram S, Luo S, Khrebtukova I, Barrette TR, Grasso C, Yu J, Lonigro RJ, Schroth G, Kumar-Sinha C, et al. Chimeric 
transcript discovery by paired-end transcriptome sequencing. Proc Natl Acad Sci U S A. 2009; 106:12353-12358.

14. Yu YP, Ding Y, Chen Z, Liu S, Michalopoulos A, Chen R, Gulzar ZG, Yang B, Cieply KM, Luvison A, Ren BG, Brooks JD, Jarrard D, et al. Novel fusion transcripts associate with progressive prostate cancer. Am J Pathol. 2014; 184:2840-2849.

15. Yoshihara K, Wang Q, Torres-Garcia W, Zheng S, Vegesna R, Kim H, Verhaak RG. The landscape and therapeutic relevance of cancer-associated transcript fusions. Oncogene. 2015; 34:4845-4854.

16. Gingeras TR. Implications of chimaeric non-co-linear transcripts. Nature. 2009; 461:206-211.

17. Nacu S, Yuan W, Kan Z, Bhatt D, Rivers CS, Stinson J, Peters BA, Modrusan Z, Jung K, Seshagiri S, Wu TD. Deep RNA sequencing analysis of readthrough gene fusions in human prostate adenocarcinoma and reference samples. BMC Med Genomics. 2011; 4:11.

18. Li H, Wang J, Mor G, Sklar J. A neoplastic gene fusion mimics trans-splicing of RNAs in normal human cells. Science. 2008; 321:1357-1361.

19. Frenkel-Morgenstern M, Lacroix V, Ezkurdia I, Levin Y, Gabashvili A, Prilusky J, Del Pozo A, Tress M, Johnson R, Guigo R, Valencia A. Chimeras taking shape: potential functions of proteins encoded by chimeric RNA transcripts. Genome Res. 2012; 22:1231-1242.

20. Jividen K, Li H. Chimeric RNAs generated by intergenic splicing in normal and cancer cells. Genes Chromosomes Cancer. 2014; 53:963-971.

21. Rickman DS, Pflueger D, Moss B, VanDoren VE, Chen CX, de la Taille A, Kuefer R, Tewari AK, Setlur SR, Demichelis F, Rubin MA. SLC45A3-ELK4 is a novel and frequent erythroblast transformation-specific fusion transcript in prostate cancer. Cancer Res. 2009; 69:2734 2738.

22. Kannan K, Wang L, Wang J, Ittmann MM, Li W, Yen L. Recurrent chimeric RNAs enriched in human prostate cancer identified by deep sequencing. Proc Natl Acad Sci U S A. 2011; 108:9172-9177.

23. Pflueger D, Terry S, Sboner A, Habegger L, Esgueva R, Lin PC, Svensson MA, Kitabayashi N, Moss BJ, MacDonald TY, Cao X, Barrette T, Tewari AK, et al. Discovery of non-ETS gene fusions in human prostate cancer using next-generation RNA sequencing. Genome Res. 2011; 21:56-67.

24. Wyatt AW, Mo F, Wang K, McConeghy B, Brahmbhatt S, Jong L, Mitchell DM, Johnston RL, Haegert A, Li E, Liew J, Yeung J, Shrestha R, et al. Heterogeneity in the inter-tumor transcriptome of high risk prostate cancer. Genome Biol. 2014; 15:426.

25. Teles Alves I, Hartjes T, McClellan E, Hiltemann S, Bottcher R, Dits N, Temanni MR, Janssen B, van Workum W, van der Spek P, Stubbs A, de Klein A, Eussen B, et al. Next-generation sequencing reveals novel rare fusion events with functional implication in prostate cancer. Oncogene. 2015; 34:568-577.

26. Luo JH, Liu S, Zuo ZH, Chen R, Tseng GC, Yu YP. Discovery and Classification of Fusion Transcripts in Prostate Cancer and Normal Prostate Tissue. Am J Pathol. 2015; 185:1834-1845.

27. Nonn L, Ananthanarayanan V, Gann PH. Evidence for field cancerization of the prostate. Prostate. 2009; 69:1470-1479.

28. Mertens F, Johansson B, Fioretos T, Mitelman F. The emerging complexity of gene fusions in cancer. Nat Rev Cancer. 2015; 15:371-381.

29. Zihni C, Mitsopoulos C, Tavares IA, Baum B, Ridley AJ, Morris JD. Prostate-derived sterile 20-like kinase 1-alpha induces apoptosis. JNK- and caspase-dependent nuclear localization is a requirement for membrane blebbing. J Biol Chem. 2007; 282:6484-6493.

30. Ingermann AR, Yang YF, Han J, Mikami A, Garza AE, Mohanraj L, Fan L, Idowu M, Ware JL, Kim HS, Lee DY, Oh Y. Identification of a novel cell death receptor mediating IGFBP-3-induced anti-tumor effects in breast and prostate cancer. J Biol Chem. 2010; 285:30233-30246.

31. Raman M, Earnest S, Zhang K, Zhao Y, Cobb MH. TAO kinases mediate activation of p38 in response to DNA damage. EMBO J. 2007; 26:2005-2014.

32. Giang DK, Cravatt BF. Molecular characterization of human and mouse fatty acid amide hydrolases. Proc Natl Acad Sci U S A. 1997; 94:2238-2242.

33. Spahr H, Habermann B, Gustafsson CM, Larsson NG, Hallberg BM. Structure of the human MTERF4-NSUN4 protein complex that regulates mitochondrial ribosome biogenesis. Proc Natl Acad Sci U S A. 2012; 109:1525315258.

34. Endsley MP, Thill R, Choudhry I, Williams CL, KajdacsyBalla A, Campbell WB, Nithipatikom K. Expression and function of fatty acid amide hydrolase in prostate cancer. Int J Cancer. 2008; 123:1318-1326.

35. Wang J, Zhao LY, Uyama T, Tsuboi K, Wu XX, Kakehi Y, Ueda N. Expression and secretion of N-acylethanolaminehydrolysing acid amidase in human prostate cancer cells. J Biochem. 2008; 144:685-690.

36. Lee SH, Hatakeyama S, Yu SY, Bao X, Ohyama C, Khoo KH, Fukuda MN, Fukuda M. Core3 O-glycan synthase suppresses tumor formation and metastasis of prostate carcinoma PC3 and LNCaP cells through downregulation of alpha2beta1 integrin complex. J Biol Chem. 2009; 284:17157-17169.

37. Crea F, Watahiki A, Quagliata L, Xue H, Pikor L, Parolia A, Wang Y, Lin D, Lam WL, Farrar WL, Isogai T, Morant R, Castori-Eppenberger $\mathrm{S}$, et al. Identification of a long noncoding RNA as a novel biomarker and potential therapeutic target for metastatic prostate cancer. Oncotarget. 2014; 5:764-774. doi: 10.18632/oncotarget.1769. 
38. Prensner JR, Chinnaiyan AM. The emergence of lncRNAs in cancer biology. Cancer Discov. 2011; 1:391-407.

39. Lindberg J, Klevebring D, Liu W, Neiman M, Xu J, Wiklund P, Wiklund F, Mills IG, Egevad L, Gronberg H. Exome sequencing of prostate cancer supports the hypothesis of independent tumour origins. Eur Urol. 2013; 63:347-353.

40. McPherson A, Hormozdiari F, Zayed A, Giuliany R, Ha G, Sun MG, Griffith M, Heravi Moussavi A, Senz J, Melnyk N, Pacheco M, Marra MA, Hirst M, et al. deFuse: an algorithm for gene fusion discovery in tumor RNA-Seq data. PLoS Comput Biol. 2011; 7:e1001138.

41. Finn RD, Bateman A, Clements J, Coggill P, Eberhardt RY, Eddy SR, Heger A, Hetherington K, Holm L, Mistry J, Sonnhammer EL, Tate J, Punta M. Pfam: the protein families database. Nucleic Acids Res. 2014; 42:D222-230.

42. Letunic I, Doerks T, Bork P. SMART: recent updates, new developments and status in 2015. Nucleic Acids Res. 2015; 43:D257-260.
43. Trapnell C, Pachter L, Salzberg SL. TopHat: discovering splice junctions with RNA-Seq. Bioinformatics. 2009; 25:1105-1111.

44. Trapnell C, Roberts A, Goff L, Pertea G, Kim D, Kelley DR, Pimentel H, Salzberg SL, Rinn JL, Pachter L. Differential gene and transcript expression analysis of RNA-seq experiments with TopHat and Cufflinks. Nat Protoc. 2012; 7:562-578.

45. Anders S, Reyes A, Huber W. Detecting differential usage of exons from RNA-seq data. Genome Res. 2012; 22:20082017.

46. Babiceanu M, Qin F, Xie Z, Jia Y, Lopez K, Janus N, Facemire L, Kumar S, Pang Y, Qi Y, Lazar IM, Li H. Recurrent chimeric fusion RNAs in non-cancer tissues and cells. Nucleic Acids Res. 2016; 44:2859-2872. 